\title{
Associations of oxytocin with metabolic parameters in obese women of childbearing age
}

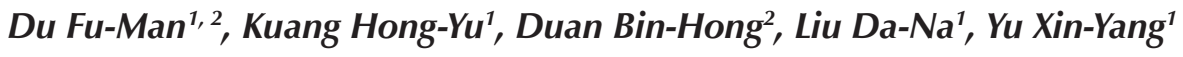 \\ ${ }^{1}$ Department of Endocrinology, First Affiliated Hospital of Harbin Medical University, Harbin, Heilongjiang Province, China \\ ${ }^{2}$ Department of Endocrinology, Heilongjiang Provincial Hospital, Harbin, Heilongjiang Province, China
}

\begin{abstract}
Introduction: The aim of this study was to compare plasma oxytocin levels in obese women of childbearing age with non-obese women of childbearing age, and to investigate the relationship between plasma oxytocin levels and metabolic parameters (including blood glucose, insulin resistance, blood lipid, and blood pressure).

Material and methods: A total of 151 obese women of childbearing age and 160 non-obese women of childbearing age were enrolled in this study. Plasma oxytocin levels were measured by electrochemiluminescence immunoassays. Height, body weight, body mass index (BMI), fasting blood glucose (FBG), fasting insulin (FI), homeostasis model assessment for insulin resistance (HOMA-IR), total triglycerides (TG), total cholesterol (TC), low-density lipoprotein-C (LDL-C), high-density lipoprotein-C (HDL-C), systolic blood pressure (SBP), and diastolic blood pressure (DBP) were measured in all subjects. Quantile regression analysis was used to analyse the associations of plasma oxytocin levels with FBG, FI, HOMA-IR, TG, TC, LDL-C, HDL-C, SBP, and DBP.

Results: In obese women of childbearing age, plasma oxytocin levels were lower compared with non-obese controls. After adjusting for age, quantile regression analysis showed that the plasma oxytocin levels were inversely associated with HOMA-IR at the quantile level between 0.27 and 0.79 (i.e. the HOMA-IR level of 2.11 and 3.07, respectively), the plasma oxytocin levels were inversely associated with TC after the quantile level of 0.21 (i.e. the TC level of 3.78 ), and the plasma oxytocin levels were inversely associated with LDL-C at all quantile levels of LDL-C. In addition, the plasma oxytocin levels showed a positive association with HDL-C at all quantile levels of HDL-C. No significant associations were found between the plasma oxytocin levels and FBG, FI, TG, SBP, and DBP.

Conclusions: Oxytocin deficiency was common in obese women of childbearing age. Oxytocin showed negative correlation with HOMA-IR, TC, and LDL-C, while it showed positive association with HDL-C. Our findings suggest that oxytocin played an important role in inhibiting metabolic disorders associated with obesity in women of childbearing age. (Endokrynol Pol 2019; 70 (5): 417-422)
\end{abstract}

Key words: oxytocin; obesity; women; childbearing; body mass index; insulin resistance; lipids; quantile regression analysis

\section{Introduction}

The worldwide upward trend in obesity has been dramatic, the prevalence of obesity has nearly tripled since 1975, and in 2016, more than 1.9 billion adults were overweight, 39\% of women aged 18 and over were overweight, according to a report by the World Health Organisation (WHO) [1]. Obesity in women of childbearing age is association with increasing risk of a number of adverse pregnancy and birth outcomes, including gestational diabetes, gestational hypertension, foetal macrosomia, miscarriage, preterm birth, birth defects, and infant mortality [2-5]. Obese women are also at greater risk than obese men of developing many chronic diseases, including metabolic syndrome, type 2 diabetes, essential hypertension, dyslipidaemia, and cardiovascular diseases [6, 7]. The common risk factors of becoming overweight or obese in women of reproductive age include order of births, pregnancy and breast-feeding triggers physiological changes, genetic and ethnic reason, socioeconomic status and abnormal endocrine function [8-10].

Oxytocin, a hypothalamic hormone that enters the peripheral circulation through the posterior pituitary, regulates a range of physiological processes, including reducing caloric intake, increasing energy expenditure, improving fat metabolism, and maintaining glucose homeostasis and insulin sensitivity [11-13]. Some studies have confirmed the association of plasma oxytocin with obesity, and oxytocin may play an important role in control of body weight in women [14]. Decreased oxytocin concentration is directly correlated with hypertension, dyslipidaemia, insulin resistance, and type 2 diabetes mellitus [15-17]. However, few studies have directly examined the associations of oxytocin with obesity in women of reproductive years because normal ovarian function and oestradiol concentration may prevent women from being obese. Nonetheless, 
some studies suggested that ovarian function is slightly lower in obese women than in healthy control subjects of young reproductive age, although ovarian reserve parameters are in normal reference range [18, 19]. In addition, some studies also revealed that lacking oestradiol can blunt oxytocin expression, decreasing circulation levels of oxytocin, and inhibiting signalling in pre-autonomic areas of the hypothalamus [20].

Therefore, we hypothesised that the plasma oxytocin may decrease in obese women of childbearing age, thus contributing to obesity, and the decreased plasma oxytocin levels may be associated with obesity-related parameters, including blood glucose, insulin resistance, blood lipids, and blood pressure.

\section{Material and methods}

\section{Subjects}

From April 2017 to October 2018, 151 obese women of childbearing age (18 to 45 years) were selected randomly from among out-patients of Heilongjiang provincial hospital, Harbin, China. 160 non-obese women controls aged $18-45$ years were selected randomly from a physical examination centre at the same time in the same hospital. Subjects with thyroid diseases, adrenal diseases, pituitary diseases, ovarian diseases, heart failure, severe hepatic or renal diseases, severe gastrointestinal diseases, malignant tumour, hypertension, diabetes mellitus, or mental illness were excluded from this study. Pregnant or lactating women were also excluded from this study.

\section{Definition}

BMI was classified into the following three categories: $18.5-23.9 \mathrm{~kg} / \mathrm{m}^{2}$ (normal weight), $24.0-27.9 \mathrm{~kg} / \mathrm{m}^{2}$ (overweight), and $\geq 28.0 \mathrm{~kg} / \mathrm{m}^{2}$ (obesity) [21]. In this study, we excluded pathological obesity caused by metabolic or endocrine diseases. In addition, women of childbearing age were those aged $18-45$ years with normal menstrual cycle.

\section{Anthropometric and laboratory measurement}

Height and weight were measured using an ultrasonic electronic height and weight measuring instrument (Umron Co., Beijing, China). Blood samples were obtained from all subjects at 8 a.m. after at least an eight-hour overnight fast. Plasma oxytocin and FI levels were determined using electrochemiluminescence immunoassays (Roche Diagnostics, Mannheim, Germany) and FPG, TG, TC, LDL-C, and HDL-C were assayed by automatic biochemical analyser (Hitachi, Ltd., Tokyo, Japan) in the Department of Experimental Diagnosis, Heilongjiang Provincial Hospital, Harbin, China. Blood pressure was measured from right arm in a sitting position after the participants had rested for 30 minutes, using a mercury sphygmomanometer. BMI was calculated according to the following formula: weight $(\mathrm{kg}) /$ height $(\mathrm{m})^{2}$.

HOMA-IR was calculated according to this formula: FI $(\mathrm{mIL} / \mathrm{L}) \times F P G(\mathrm{mmol} / \mathrm{L}) / 22.5$.

\section{Medical ethical approval}

This study was approved by the medical Ethics Committee of Heilongjiang Provincial Hospital. Written consent to participate in this study was obtained from all participants. Only those who signed the consent form were enrolled in the study. All study protocols were carried out in accordance with the declaration of Helsinki [22].

\section{Statistical analysis}

Statistical Package for the Social Sciences, version 22.0 (SPSS Inc., Chicago, IL, USA) was used for statistical analysis. The general variables of the subjects across the two study groups (i.e. the obese women group and the non-obese women group) were expressed as means and standard deviations. The variables were compared using two-sample $t$-test. Differences were considered significant at $\mathrm{p}<0.05$ for two sides. Quantile regression analysis [23] was used to analyse the associations of plasma oxytocin levels with obesityrelated parameters in two groups. The regression coefficients of the variable (plasma oxytocin) in the quantile regression model differ across the varying quantile levels of FPG, FI, HOMA-IR, TC, TG, LDL-C, HDL-C, SBP, and DBP. The figures in this study were drawn using GraphPad Prism version 7 (GraphPad Software, Inc., La Jolla, CA, USA).

\section{Results}

\section{Clinical and laboratory characteristics of the two study groups}

From all 311 participants, there were 151 subjects $(48.55 \%)$ in the obese women group and 160 subjects $(51.45 \%)$ in the non-obese women group. The descriptive statistics of the characteristics are shown in Table I. The obese women group had higher BMI $(\mathrm{p}<0.001)$, higher FPG $(p=0.005)$, higher TC $(p<0.001)$, higher LDL-C ( $\mathrm{p}<0.001)$, higher SBP $(\mathrm{P}=0.008)$, lower oxytocin ( $p<0.001)$, and lower HDL-C ( $p<0.001)$ levels compared with the non-obese women group. As for all the other characteristics, including age, $\mathrm{FI}$, and DBP, the differences between two groups did not reach statistical significance $(\mathrm{p}>0.05)$.

\section{Associations of plasma oxytocin levels with obesity-related parameters}

Table II shows the coefficient estimation with 95\% CI for the associations of plasma oxytocin levels with HOMA-IR, TC, LDL-C, and HDL-C at varying quantile levels of these variables. After adjusting for age, quantile regression analysis showed that the plasma oxytocin levels were inversely associated with HOMA-IR at the quantile level between 0.27 and 0.79 (i.e. the HOMA-IR level of 2.11 and 3.07, respectively), the plasma oxytocin levels were inversely associated with TC after the quantile level of 0.21 (i.e. the TC level of 3.78 ), and the plasma oxytocin levels were inversely associated with LDL-C at all quantile levels of LDL-C. In addition, the plasma oxytocin levels showed a positive association with HDL-C at all quantile levels of HDL-C. No significant associations were found between plasma oxytocin levels and FBG, FI, TG, SBP, and DBP.

The plot in Figures 1-4 shows the coefficients for the associations of plasma oxytocin levels with HOMA-IR, TC, LDL-C, and HDL-C across the selected quantile levels of these variables. The grey area represents the $95 \%$ CI of the coefficients, the black curve represents the estimated coefficients, and the dotted line represents 
Table I. Comparison of clinical and laboratory characteristics between obese women and non-obese women

\begin{tabular}{|c|c|c|c|c|}
\hline Characteristics & $\begin{array}{c}\text { Total } \\
(\mathrm{n}=\mathbf{3 1 1})\end{array}$ & $\begin{array}{l}\text { Non-obese women group } \\
\qquad(\mathrm{n}=160)\end{array}$ & $\begin{array}{l}\text { Obese women group } \\
\qquad(\mathrm{n}=151)\end{array}$ & p-value \\
\hline Age [years] & $32.72 \pm 7.37$ & $32.74 \pm 7.04$ & $32.70 \pm 7.72$ & 0.966 \\
\hline BMI [kg/m²] & $25.89 \pm 3.93$ & $22.51 \pm 2.20$ & $29.48 \pm 1.29^{*}$ & $<0.001$ \\
\hline FPG [mmol/L] & $5.01 \pm 0.61$ & $4.91 \pm 0.59$ & $5.11 \pm 0.62^{*}$ & 0.005 \\
\hline $\mathrm{FI}[\mu \mathrm{U} / \mathrm{mL}]$ & $11.41 \pm 2.24$ & $11.46 \pm 2.15$ & $11.36 \pm 2.33$ & 0.703 \\
\hline HOMA-IR & $2.54 \pm 0.58$ & $2.50 \pm 0.57$ & $2.57 \pm 0.59$ & 0.284 \\
\hline TG $[\mathrm{mmol} / \mathrm{L}]$ & $1.89 \pm 0.57$ & $1.87 \pm 0.41$ & $1.91 \pm 0.70$ & 0.486 \\
\hline $\mathrm{TC}[\mathrm{mmol} / \mathrm{L}]$ & $4.68 \pm 1.02$ & $4.29 \pm 0.68$ & $5.09 \pm 1.15^{*}$ & $<0.001$ \\
\hline LDL-C [mmol/L] & $2.17 \pm 0.77$ & $1.78 \pm 0.52$ & $2.58 \pm 0.77^{*}$ & $<0.001$ \\
\hline $\mathrm{HDL}[\mathrm{mmol} / \mathrm{L}]$ & $1.38 \pm 0.39$ & $1.55 \pm 0.40$ & $1.19 \pm 0.29^{*}$ & $<0.001$ \\
\hline $\mathrm{DBP}$ [mm Hg] & $70.23 \pm 8.03$ & $69.64 \pm 7.68$ & $70.87 \pm 8.36$ & 0.177 \\
\hline SBP [mm Hg] & $113.13 \pm 10.74$ & $111.55 \pm 11.00$ & $114.79 \pm 10.23^{*}$ & 0.008 \\
\hline Oxytocin [ng/mL] & $2.52 \pm 0.82$ & $2.96 \pm 0.51$ & $2.04 \pm 0.82^{*}$ & $<0.001$ \\
\hline
\end{tabular}

$\mathrm{BMI}$ — body mass index; FPG — fasting plasma glucose; FI — fasting plasma insulin; HOMA-IR — homeostatic model assessment for insulin resistance; TG — triglyceride; TC — total cholesterol; LDL-C — low-density lipoprotein cholesterol; HDL — high-density lipoprotein; DBP — diastolic blood pressure; SBP — systolic blood pressure. Variables are expressed as means and standard deviations. "significantly different $(p<0.05)$ from the non-obese women group

Table II. Associations of plasma oxytocin levels with HOMA-IR, TC, LDL-C, and HDL-C at the selected quantile levels of these variables (coefficients and $95 \%$ CI)

\begin{tabular}{|c|c|c|c|c|c|c|c|c|}
\hline \multirow{2}{*}{$\mathbf{Q} \mathbf{R}^{\dagger}$} & \multicolumn{2}{|c|}{ HOMA-IR } & \multicolumn{2}{|c|}{ TC } & \multicolumn{2}{|c|}{ LDL-C } & \multicolumn{2}{|c|}{ HDL-C } \\
\hline & Coefficients & $95 \% \mathrm{CI}$ & Coefficients & $95 \% \mathrm{Cl}$ & Coefficients & $95 \% \mathrm{Cl}$ & Coefficients & $95 \% \mathrm{CI}$ \\
\hline 0.1 & 0.013 & $-0.081,0.067$ & 0.002 & $-0.177,0.144$ & -0.305 & $\begin{array}{l}-0.396 \\
-0.132\end{array}$ & 0.076 & $0.044,0.135$ \\
\hline 0.2 & -0.007 & $-0.120,0.041$ & -0.237 & $\begin{array}{l}-0.403 \\
-0.042\end{array}$ & -0.206 & $\begin{array}{l}-0.303 \\
-0.109\end{array}$ & 0.114 & $0.068,0.131$ \\
\hline 0.3 & -0.101 & $\begin{array}{l}-0.161, \\
-0.015\end{array}$ & -0.383 & $\begin{array}{l}-0.508 \\
-0.141\end{array}$ & -0.163 & $\begin{array}{l}-0.342 \\
-0.048\end{array}$ & 0.122 & $0.089,0.169$ \\
\hline 0.4 & -0.125 & $\begin{array}{l}-0.214 \\
-0.062\end{array}$ & -0.388 & $\begin{array}{l}-0.605 \\
-0.232\end{array}$ & -0.206 & $\begin{array}{l}-0.325 \\
-0.079\end{array}$ & 0.139 & $0.065,0.200$ \\
\hline 0.5 & -0.182 & $\begin{array}{l}-0.240 \\
-0.074\end{array}$ & -0.395 & $\begin{array}{l}-0.553 \\
-0.240\end{array}$ & -0.289 & $\begin{array}{l}-0.357 \\
-0.176\end{array}$ & 0.145 & $0.073,0.230$ \\
\hline 0.6 & -0.164 & $\begin{array}{l}-0.240 \\
-0.078\end{array}$ & -0.374 & $\begin{array}{l}-0.532 \\
-0.186\end{array}$ & -0.323 & $\begin{array}{l}-0.420 \\
-0.226\end{array}$ & 0.181 & $0.071,0.246$ \\
\hline 0.7 & -0.122 & $\begin{array}{l}-0.213 \\
-0.057\end{array}$ & -0.417 & $\begin{array}{l}-0.612 \\
-0.257\end{array}$ & -0.342 & $\begin{array}{l}-0.433 \\
-0.243 \\
\end{array}$ & 0.182 & $0.117,0.293$ \\
\hline 0.8 & -0.113 & $-0.296,0.010$ & -0.537 & $\begin{array}{l}-0.674 \\
-0.420\end{array}$ & -0.332 & $\begin{array}{l}-0.507 \\
-0.259\end{array}$ & 0.142 & $0.036,0.295$ \\
\hline 0.9 & -0.116 & $\begin{array}{l}-0.178 \\
-0.131\end{array}$ & -0.506 & $\begin{array}{l}-0.620 \\
-0.412\end{array}$ & -0.467 & $\begin{array}{l}-0.557 \\
-0.293\end{array}$ & 0.133 & $0.020,0.188$ \\
\hline
\end{tabular}

HOMA-IR — homeostatic model assessment for insulin resistance; TC — total cholesterol; LDL-C — low-density lipoprotein cholesterol; HDL — high-density lipoprotein; $\mathrm{Cl}$ - confidence interval; ${ }^{T}$ The quantile levels ranged from 0.1 to 0.9 , in which the corresponding values of HOMA-IR were 1.82, 1.98, 2.15, 2.32, 2.49, 2.64, 2.80, 3.08, and 3.38; the corresponding values of TC were 3.37, 3.77, 4.09, 4.35, 4.53, 4.86, 5.11, 5.48, and 6.00; the corresponding values of LDL-C were 1.33, $1.65,1.79,1.97,2.02,2.12,2.30,2.76$, and 3.14 ; and the corresponding values of $H D L$ were $0.94,1.03,1.12,1.21,1.31,1.43,1.54,1.73$, and 1.93 , respectively

the coefficient $=0$. We found the association between plasma oxytocin levels and HOMA-IR at the quantile level between 0.27 and 0.79 (i.e. the HOMA-IR levels of 2.11 and 3.07, respectively). An inverse association of plasma oxytocin levels with TC was found after the quantile level of 0.21 (i.e. the TC level of 3.78), and it became stronger after that level. The inverse association of plasma oxytocin levels with LDL-C was seen at all quantile levels and became stronger after the quantile level of 0.32 (i.e. the LDL level of 1.91). The plasma oxytocin levels showed a positive association with HDL-C at all quantile levels of HDL-C, and the correlation was 


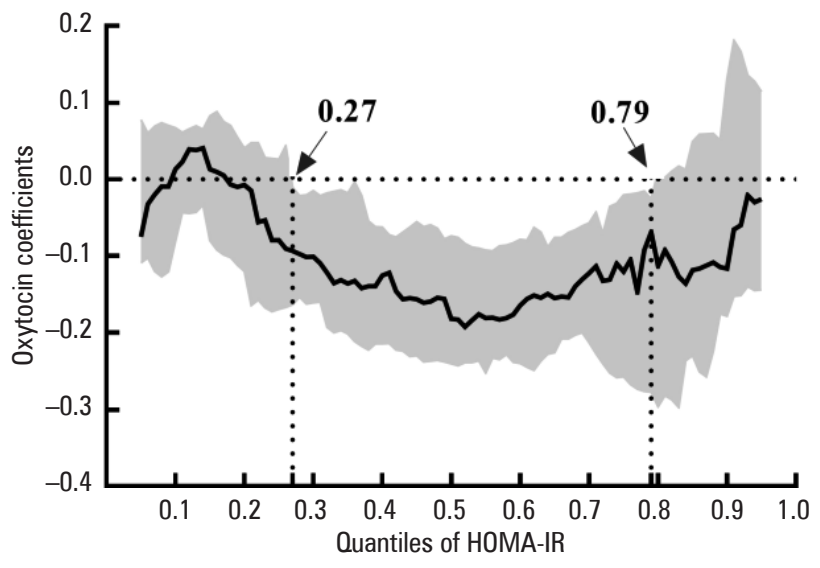

Figure 1. Coefficients of oxytocin for the associations of plasma oxytocin levels and HOMA-IR across the selected quantile levels of HOMA-IR. HOMA-IR - homeostatic model assessment for insulin resistance

The quantile levels ranged from 0.1 to 0.9 , in which the corresponding values of HOMA-IR were $1.82,1.98,2.15,2.32,2.49,2.64,2.80,3.08$, and 3.38, respectively

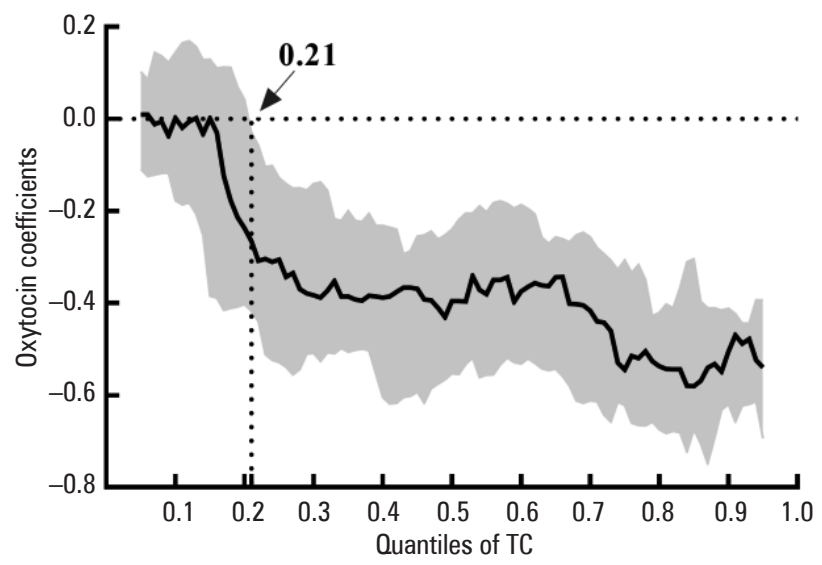

Figure 2. Coefficients of oxytocin for the associations of plasma oxytocin levels and TC across the selected quantile levels of TC. TC - total cholesterol

The quantile levels ranged from 0.1 to 0.9 , in which the corresponding values of TC were $3.37,3.77,4.09,4.35,4.53,4.86,5.11,5.48$, and 6.00 , respectively

strongest at the quantile level of 0.62 (i.e. the HDL-C level of 1.48).

\section{Discussion}

The purpose of this study was to examine the plasma oxytocin levels in obese women of childbearing age, and to investigate the relationship between plasma oxytocin levels and metabolic parameters. In this study, we included FPG, FI, HOMA-IR, TC, TG, LDL-C, HDL-C, $\mathrm{SBP}$, and DBP as evaluation indices of metabolism related to obesity.

We demonstrated that the obese women of reproductive age had lower plasma oxytocin levels

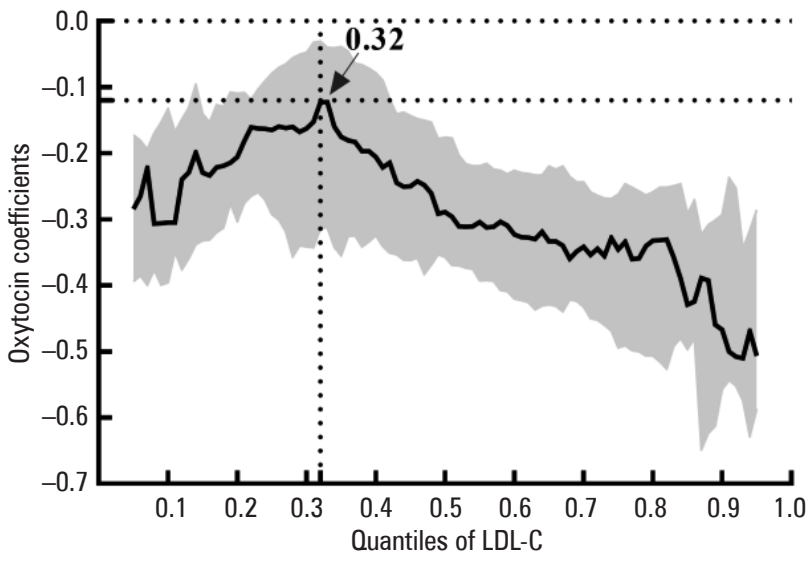

Figure 3. Coefficients of oxytocin for the associations of plasma oxytocin levels and LDL-C across the selected quantile levels of LDL-C. LDL-C - low-density lipoprotein cholesterol

The quantile levels ranged from 0.1 to 0.9 , in which the corresponding values of $L D L-C$ were $1.33,1.65,1.79,1.97,2.02,2.12,2.30,2.76$, and 3.14, respectively

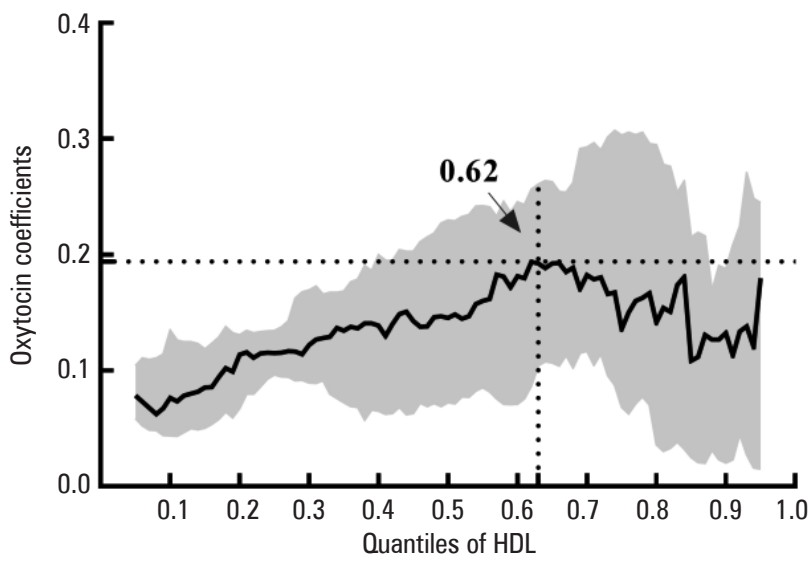

Figure 4. Coefficients of oxytocin for the associations of plasma oxytocin levels and HDL-C across the selected quantile levels of HDL-C. HDL — high-density lipoprotein

The quantile levels ranged from 0.1 to 0.9 , in which the corresponding values of HDL were $0.94,1.03,1.12,1.21,1.31,1.43,1.54,1.73$, and 1.93 , respectively

compared with non-obese women of the same age. This result was in agreement with some previous reports. However, most of the related studies were done on postmenopausal women or women after ovariectomy $[14,24]$. Oxytocin is an anorexigenic neuropeptide that controls metabolic homeostasis mainly via regulating food intake and energy expenditure [25]. Laboratory animal experiments also confirmed that oxytocin can increase brown adipose tissue thermogenesis [26-28]. Despite the basic animal research showing beneficial effects of oxytocin on adjusting metabolism and losing weight, clinical studies on oxytocin's therapeutic potential in human obesity are still in their early stages $[11,29,30]$. 
In this study, after adjusting for age, we found that the plasma oxytocin levels were inversely associated with HOMA-IR, LC, and LDL-C, and it also showed an positive association with HDL-C. Moreover, we found that the inverse association between plasma oxytocin levels and HOMA-IR was seen above the HOMA-IR level of 2.11, and the inverse association of plasma oxytocin levels with TC was found above the TC level of 3.78 and became stronger above that level. We also found that the inverse association of plasma oxytocin levels with LDL-C was seen at all LDL-C levels and became stronger above the LDL level of 1.91. The plasma oxytocin levels showed a positive association with HDL-C at all levels of HDL-C, and the correlation was strongest at HDL-C level of 1.48.

These results suggest for the first time that oxytocin played an important role in inhibiting obesity-related metabolic disorders, including insulin resistance and dyslipidaemia, and the association between oxytocin and dyslipidaemia was more significant. Furthermore, the higher the TC and LDL-C levels, the more significant the correlations. In this study, we selected obese women in reproductive age as the research group after excluding pathological obesity caused by metabolic or endocrine diseases. These research subjects had normal ovarian function and menstrual cycle. Therefore, the differences between the variables of the two groups were small, and the association between them may be weak. Hence, quantile regression analysis was used in this study. The regression coefficients of the variables in the quantile regression model differ across the selected quantile levels of dependent variables, which is very useful to investigate the associations between the variables in which there may be only weak associations between the variables.

A previous study revealed that oxytocin showed negative correlation with FI and HOMA in obese women with polycystic ovary syndrome [31]. Another study showed that oxytocin concentration correlated negatively with glycated haemoglobin $\left(\mathrm{HbA}_{1 \mathrm{c}}\right)$ and FPG in diabetic metabolic syndrome patients [32]. In this study, we failed to observe an obvious relationship between oxytocin and serum insulin levels and blood sugar levels. Such results may be due to the different subjects included in the study. So far, few studies have examined the relationship between oxytocin and blood lipid or lipoprotein, and the findings have been inconsistent; in some of them there were no statistically significant relationships between oxytocin and lipid levels $[17,33,34]$. One of the studies investigated the associations between oxytocin and glycolipid metabolism in type 2 diabetes patients and obtained ideal conclusions. They found that serum oxytocin levels were negatively correlated with TC, TG, and LDL-C [17].
Because the metabolic disorders in diabetes patients are more obvious, it is easier to gain a positive conclusion in research on the correlation between oxytocin and metabolic parameters. In our study, the difference in metabolic parameters between obese women of childbearing age and non-obese women was not significant, especially blood glucose and blood pressure, so we did not draw corresponding positive conclusions.

One limitation of our study was that the sample size was small. A larger cohort is needed to investigate plasma oxytocin levels in obese women of reproductive age and their relationship with metabolic parameters. Another limitation was that the gonadal axis and its association with oxytocin were not evaluated in this study. In females, oxytocin secretion is affected by the function of the ovaries $[20,35]$. One study showed that $17 \beta$-oestradiol increased expression of oxytocin mRNA in the paraventricular nucleus of the hypothalamus and then increased plasma oxytocin protein levels [36]. In the future, further studies are needed to expand the study variables in order to obtain more reliable conclusions.

In this study, the results suggest that oxytocin plays an important role in inhibiting metabolic disorders. Therefore, our future implementation of oxytocin-based studies will focus on its therapeutic effects on controlling body weight, energy balance, glucolipid metabolism, and insulin resistance. Moreover, early studies have looked at changes in plasma oxytocin levels after weight loss. It was found that plasma levels of oxytocin were almost four-fold higher in obese people after weight reduction following gastric banding [37]. However, few studies have investigated the effects of weight-loss drugs on levels of oxytocin. Future research will also look at the effects of some anti-obesity drugs on oxytocin levels.

\section{Conclusions}

Oxytocin deficiency was common in obese women of childbearing age. Oxytocin showed a negative correlation with HOMA-IR, TC, and LDL-C, while it showed a positive association with HDL-C. Furthermore, the higher the TC and LDL-C levels, the more significant the correlations. Our findings suggested that oxytocin played an important role in inhibiting metabolic disorders associated obesity in women of childbearing age.

\section{Conflicts of interest}

All authors have no conflicts of interest to declare in relation to this article.

\section{Funding agency}

This study received funding support from the Health Commission of Heilongjiang Province (Research project No. 2016-504). 


\section{References}

1. Barich F, Zahrou FE, Laamiri FZ, et al. Association of Obesity and Socioeconomic Status among Women of Childbearing Age Living in Urban Area of Morocco. J Nutr Metab. 2018; 2018: 6043042, doi: 10.1155/2018/6043042, indexed in Pubmed: 30151280.

2. Broughton DE, Moley KH. Obesity and female infertility: potential mediators of obesity's impact. Fertil Steril. 2017; 107(4): 840-847, doi: 10.1016/j. fertnstert.2017.01.017, indexed in Pubmed: 28292619.

3. Declercq E, MacDorman M, Cabral H, et al. Prepregnancy Body Mass Index and Infant Mortality in 38 U.S. States, 2012-2013. Obstet Gynecol. 2016; 127(2): 279-287, doi: 10.1097/AOG.0000000000001241, indexed in Pubmed: 26942355.

4. Stang J, Huffman LG. Position of the Academy of Nutrition and Dietetics: Obesity, Reproduction, and Pregnancy Outcomes. J Acad Nutr Diet. 2016; 116(4): 677-691, doi: 10.1016/j.jand.2016.01.008, indexed in Pubmed: 27017177.

5. Walzem RL, Chen Se. Obesity-induced dysfunctions in female reproduction: lessons from birds and mammals. Adv Nutr. 2014; 5(2): 199-206, doi: 10.3945/an.113.004747, indexed in Pubmed: 24618762.

6. Pucci G, Alcidi R, Tap L, et al. Sex- and gender-related prevalence, cardiovascular risk and therapeutic approach in metabolic syndrome: A review of the literature. Pharmacol Res. 2017; 120: 34-42, doi: 10.1016/j. phrs.2017.03.008, indexed in Pubmed: 28300617.

7. Appelman Y, van Rijn BB, Ten Haaf ME, et al. Sex differences in cardiovascular risk factors and disease prevention. Atherosclerosis. 2015; 241(1): 211-218, doi: 10.1016/j.atherosclerosis.2015.01.027, indexed in Pubmed: 25670232.

8. National Center for Health Statistics (US). Health, United States, 2015: with Special Feature on Racial and Ethnic Health Disparities. National Center for Health Statistics (US), Hyattsville, MD 2016: Report No: 2016-1232.-1232.

9. O'Connor SG, Habre R, Bastain TM, et al. Within-subject effects of environmental and social stressors on pre- and post-partum obesity-related biobehavioral responses in low-income Hispanic women: protocol of an intensive longitudinal study. BMC Public Health. 2019; 19(1): 253, doi: 10.1186/s12889-019-6583-x, indexed in Pubmed: 30819155.

10. Siega-Riz AM. Prepregnancy obesity: determinants, consequences, and solutions. Adv Nutr. 2012; 3(1): 105-107, doi: 10.3945/an.111.001081, indexed in Pubmed: 22332108.

11. Lawson EA. The effects of oxytocin on eating behaviour and metabolism in humans. Nat Rev Endocrinol. 2017; 13(12): 700-709, doi: 10.1038/nrendo.2017.115, indexed in Pubmed: 28960210

12. Blevins JE, Graham JL, Morton GJ, et al. Chronic oxytocin administration inhibits food intake, increases energy expenditure, and produces weight loss in fructose-fed obese rhesus monkeys. Am J Physiol Regul Integr Comp Physiol. 2015; 308(5): R431-R438, doi: 10.1152/ajpregu.00441.2014, indexed in Pubmed: 25540103.

13. Spetter MS, Hallschmid M. Current findings on the role of oxytocin in the regulation of food intake. Physiol Behav. 2017; 176: 31-39, doi: 10.1016/j. physbeh.2017.03.007, indexed in Pubmed: 28284882

14. Maestrini S, Mele C, Mai S, et al. Plasma Oxytocin Concentration in Pre- and Postmenopausal Women: Its Relationship with Obesity, Body Composition and Metabolic Variables. Obes Facts. 2018; 11(5): 429-439, doi: 10.1159/000492001, indexed in Pubmed: 30372704.

15. Jameson $\mathrm{H}$, Bateman $\mathrm{R}$, Byrne $\mathrm{P}$, et al. Oxytocin neuron activation prevents hypertension that occurs with chronic intermittent hypoxia/hypercapnia in rats. Am J Physiol Heart Circ Physiol. 2016; 310(11): H1549-H1557, doi: 10.1152/ajpheart.00808.2015, indexed in Pubmed: 27016581.

16. Tofiq KO, Bulatova N, Kasabri V, et al. Increased lipocalin-2 vs reduced oxytocin in relation with adiposity, atherogenicity and hematological indices in metabolic syndrome patients with and without prediabetes. Bratisl Lek Listy. 2018; 119(12): 762-769, doi: 10.4149/BLL_2018_139, indexed in Pubmed: 30686015.

17. Qian W, Zhu T, Tang B, et al. Decreased circulating levels of oxytocin in obesity and newly diagnosed type 2 diabetic patients. J Clin Endocrinol Metab. 2014; 99(12): 4683-4689, doi: 10.1210/jc.2014-2206, indexed in Pubmed: 25233153.

18. Grigoryan OR, Mikheev RK, Andreeva EN, et al. Comparative analysis of ovarian reserve in women with obesity in reproductive period. Ter Arkh. 2018; 90(10): 30-34, doi: 10.26442/terarkh2018901030-34, indexed in Pubmed: 30701792.

19. Michalakis K, Mintziori G, Kaprara A, et al. The complex interaction between obesity, metabolic syndrome and reproductive axis: a narrative review. Metabolism. 2013; 62(4): 457-478, doi: 10.1016/j. metabol.2012.08.012, indexed in Pubmed: 22999785.

20. De Melo VU, Saldanha RRM, Dos Santos CR, et al. Ovarian Hormone Deprivation Reduces Oxytocin Expression in Paraventricular Nucleus Preautonomic Neurons and Correlates with Baroreflex Impairment in Rats. Front Physiol. 2016; 7: 461, doi: 10.3389/fphys.2016.00461, indexed in Pubmed: 27790154.

21. Zhou B. Coorperative Meta-Analysis Group Of China Obesity Task Force. [Predictive values of body mass index and waist circumference to risk factors of related diseases in Chinese adult population] Zhonghua Liu Xing Bing Xue Za Zhi. 2002; 23(1): 5-10, indexed in Pubmed: 12015100.

22. World Medical Association. World Medical Association Declaration of Helsinki: ethical principles for medical research involving human subjects. JAMA. 2013; 310(20): 2191-2194, doi: 10.1001/jama.2013.281053, indexed in Pubmed: 24141714.

23. Wei Y, Pere A, Koenker R, et al. Quantile regression methods for reference growth charts. Stat Med. 2006; 25(8): 1369-1382, doi: 10.1002/sim.2271, indexed in Pubmed: 16143984

24. Beranger GE, Pisani DF, Castel J, et al. Oxytocin reverses ovariectomy-induced osteopenia and body fat gain. Endocrinology. 2014; 155(4): 1340-1352, doi: 10.1210/en.2013-1688, indexed in Pubmed: 24506069.

25. Blevins JE, Ho JM. Role of oxytocin signaling in the regulation of body weight. Rev Endocr Metab Disord. 2013; 14(4): 311-329, doi: 10.1007/s11154-013-9260-x, indexed in Pubmed: 24065622.

26. Morton GJ, Thatcher BS, Reidelberger RD, et al. Peripheral oxytocin suppresses food intake and causes weight loss in diet-induced obese rats. Am J Physiol Endocrinol Metab. 2012; 302(1): E134-E144, doi: 10.1152/ajpendo.00296.2011, indexed in Pubmed: 22008455.

27. Deblon N, Veyrat-Durebex C, Bourgoin L, et al. Mechanisms of the anti-obesity effects of oxytocin in diet-induced obese rats. PLoS One. 2011; 6(9): e25565, doi: 10.1371/journal.pone.0025565, indexed in Pubmed: 21980491.

28. Roberts ZS, Wolden-Hanson T, Matsen ME, et al. Chronic hindbrain administration of oxytocin is sufficient to elicit weight loss in diet-induced obese rats. Am J Physiol Regul Integr Comp Physiol. 2017; 313(4): R357-R371, doi: 10.1152/ajpregu.00169.2017, indexed in Pubmed: 28747407

29. Olszewski PK, Klockars A, Levine AS. Oxytocin and potential benefits for obesity treatment. Curr Opin Endocrinol Diabetes Obes. 2017; 24(5): 320-325, doi: 10.1097/MED.0000000000000351, indexed in Pubmed: 28590323.

30. Blevins JE, Baskin DG. Translational and therapeutic potential of oxytocin as an anti-obesity strategy: Insights from rodents, nonhuman primates and humans. Physiol Behav. 2015; 152(Pt B): 438-449, doi: 10.1016/j. physbeh.2015.05.023, indexed in Pubmed: 26013577.

31. Jahromi BN, Dabbaghmanesh MH, Bakhshaie P, et al. Assessment of oxytocin level, glucose metabolism components and cutoff values for oxytocin and anti-mullerian hormone in infertile PCOS women. Taiwan J Obstet Gynecol. 2018; 57(4): 555-559, doi: 10.1016/j.tjog.2018.06.015, indexed in Pubmed: 30122578.

32. Al-Rawashdeh A, Kasabri V, Bulatova N, et al. The correlation between plasma levels of oxytocin and betatrophin in non-diabetic and diabetic metabolic syndrome patients: A cross sectional study from Jordan. Diabetes Metab Syndr. 2017; 11(1): 59-67, doi: 10.1016/j.dsx.2016.08.008, indexed in Pubmed: 27578619.

33. Szulc P, Amri EzZ, Varennes A, et al. High serum oxytocin is associated with metabolic syndrome in older men - The MINOS study. Diabetes Res Clin Pract. 2016; 122: 17-27, doi: 10.1016/j.diabres.2016.09.022, indexed in Pubmed: 27764720

34. Binay Ç, Paketçi C, Güzel S, et al. Serum Irisin and Oxytocin Levels as Predictors of Metabolic Parameters in Obese Children. J Clin Res Pediatr Endocrinol. 2017; 9(2): 124-131, doi: 10.4274/jcrpe.3963, indexed in Pubmed: 28077341.

35. Lucio-Oliveira F, Franci CR. Effect of the interaction between food state and the action of estrogen on oxytocinergic system activity. J Endocrinol. 2012; 212(2): 129-138, doi: 10.1530/JOE-11-0272, indexed in Pubmed: 22083216

36. Sloan DK, Spencer DS, Curtis KS. Estrogen effects on oxytocinergic pathways that regulate food intake. Horm Behav. 2018; 105: 128-137, doi: 10.1016/j.yhbeh.2018.08.007, indexed in Pubmed: 30118729.

37. Stock S, Granström L, Backman L, et al. Elevated plasma levels of oxytocin in obese subjects before and after gastric banding. Int J Obes. 1989; 13(2): 213-222, indexed in Pubmed: 2744933. 